Elsevier Editorial System(tm) for

Biochemical and Biophysical Research Communications

Manuscript Draft

Manuscript Number: BBRC-19-8549

Title: Pseudouridines or how to draw on weak energy differences

Article Type: SI: BBRC 60th Anniversary

Keywords: RNA

Corresponding Author: Professor Eric Westhof,

Corresponding Author's Institution: U.P.R. 9002

First Author: Eric Westhof

Order of Authors: Eric Westhof 
This article is submitted for the BBRC $60^{\text {th }}$ Anniversary Special Issue, by Michael Lacy on behalf of the
author. This article is submitted for the BBRC $60^{\text {th }}$ Anniversary Special Issue, by Michael Lacy on behalf of the
author.

Cover Letter

.
This article is submitted for the BBRC $60^{\text {th }}$ Anniversary Special Issue, by Michael Lacy on behalf of the
author.

This article is submitted for the BBRC $60^{\text {th }}$ Anniversary Special Issue, by Michael Lacy on behalf of the
author. 


\title{
Pseudouridines or how to draw on weak energy differences
}

\author{
Eric Westhof \\ Architecture et Réactivité de l'ARN, Université de Strasbourg, Institut de Biologie \\ Moléculaire et Cellulaire du CNRS, 15 rue René Descartes, 67084 Strasbourg, France.
}

\begin{abstract}
In many RNA molecules, pseudouridines occur at conserved positions in functional sites. A great diversity of pseudouridine synthases guarantees the insertion of the modified base at precise locations. The accepted structural role of pseudouridines is a reduction of the RNA flexibility around the modification site. However, experiments rarely yield clear-cut evidence. The article "Dynamic stacking of an expected branch point adenosine in duplexes containing pseudouridine-modified or unmodified U2 snRNA sites" published in 2019 in Biochemical and Biophysical Research Communication by Kennedy et al. constitute a provocative case [1]. This example illustrates how a definite conformational state can be selected through small energy differences in a constrained environment.
\end{abstract}

\section{Structural characteristics of pseudouridines}

Pseudouridine $(\psi)$, a carbon-carbon ribonucleoside of an isomer of uracil, is the most frequent modified residue in several RNAs with diverse but central functions in all kingdoms of life (for recent reviews, please see [2-4]. Pseudouridine modified ribosomal RNAs and transfer RNAs are prerequisite for efficient ribosomal assembly and decoding. The spliceosomal RNAs contain also pseudouridines at functional sites. Pseudouridines are inserted into RNAs by pseudouridine synthases either by enzymes alone or by RNA-protein complexes in which the RNA guide to the site of modification. There are 13 pseudouridine synthases [4] in human cells and about 200 guide RNA sequences [5] (called H/ACA box snoRNAs).

Structurally, pseudouridines favor the helical and stacked conformation of nucleotides (with C3'-endo riboses and the anti orientation of the base) [6]. Within a polynucleotide, this conformation is further stabilized by a water molecule linking the additional available nitrogen on the ring to anionic phosphate oxygens of the 5'-phosphate groups [7-10] (see Figure 1). Because of the right-handedness of RNA helices, the 3'-end nucleotide stacks well 
with the preceding nucleotide but not the 5 '-end one. To stabilize a helical stem it is therefore better to increase at the 5'-helical end the stacking (e.g. by a G nucleotide) and both the stacking and helical properties (by a $\psi$ nucleotide). Within a helical stem or in internal loops, the situation may be more difficult to assess. Although data on tRNAs show that pseudouridines localize to regions where they contribute to the stabilization of specific RNA folds, this is not the case in many other situations. And there are still many occurrences where pseudouridines appear as "a natural analogue of uridine of unpredictable properties" [11] (cited in [8]).

\section{The example of U2 snRNA}

One such example is a short segment of U2 snRNA that during splicing anneals with the intronic branch point sequence leading to the extrusion of the reacting adenosine leading to the formation of the $2^{\prime}-5^{\prime}$ branch intron lariat [12]. In the duplex between U2 and the branch point sequence, the pseudouridine in $\mathrm{U} 2$ faces two nucleotides (A $\underline{\mathrm{A}}$ in yeast but NA in humans where the reactive $A$ is underlined [13]). NMR data showed how the pseudouridine alters the dynamical landscape of the duplex promoting the extra-helical conformation of the reactive A [14]. However, crystal structures showed alternative conformers [9]. This is further emphasized by the NMR data presented in the recent Biochemical and Biophysical Research Communication article from Kennedy and colleagues [1]. They studied the case with GA facing the pseudouridine. The pseudouridine makes a Watson-Crick pair with the $\underline{A}$ and the $G$ is extra-helically nudged in the shallow minor groove with a syn conformation [1]. In such a conformation the $\mathrm{O}^{\prime}$ ' $(\underline{\mathrm{A}})$ is not available for forming the first step of splicing and an alternative conformation ought to be stabilized within the spliceosome [1]. One can argue that, although formation of Watson-Crick $(\psi-\mathrm{A} v s \mathrm{U}-\mathrm{A})$ or wobble $(\psi-\mathrm{G} v s$ UoG) pairs with $\psi$ enhances thermal stabilities compared to $\mathrm{U}$, a wobble pair is generally weaker than a Watson-Crick one [15], which may in part explain the observations. The contribution of the pseudouridine is therefore not sufficient for promoting the reactive conformation. It is indeed frequently observed in various systems that the comparative effects in presence of $\psi$ or $U$ are rarely conclusive and point to variations in the equilibrium populations of conformational states. The major conclusion stays that the pseudouridine modification "reduces the flexibility of the RNA in the vicinity of the modification" [16]. 


\section{Conformational state selection in a constrained environment}

Now, the spliceosome is an incredibly complex machinery at all stages of assembly and along the reaction path with five snRNAs (U1, U2, U4, U5, U6) and about one hundred protein cofactors [17]. The structural constraints due to the dynamical compaction during assembly, together with the targeted actions of helicase and chaperone proteins, can influence the selection of precise and well-defined conformations. Within that constrained environment, minor energetic differences synergize to orient the selection to definite conformational states. Or, in other words, the constrained folds amplify small energy differences by selective capture of conformations. In the absence of selective pressure, the conformational states exchange dynamically, blurring the visualization of clear-cut effects. At $\psi$ nucleotides, the small energy bias for the preferred helical state, together with the capture of a inter-nucleotide bridging water molecule, drives in a constrained environment the conformational state selection.

Similarly, for efficient and smooth decoding at the ribosomal A site, the anticodon loop of the incoming aminoacylated tRNA should be highly populated in a conformation where the anticodon triplet in a stacked helical arrangement for base pairing with the mRNA codon. The surrounding nucleotides of the anticodon loop contribute to the dynamics of the loop. Nucleotide modifications promoting the pre-organization of the loop for pairing with the codon, especially at the 3'-end of the anticodon loop, will sustain translation efficiently. The contributions of such modifications (e.g. $\psi 38$ or $\psi 39[8,16])$ will be critically important when the energy of the codon-anticodon triplet is AU-rich and weak [18] or contains non-WatsonCrick pairs $[19,20]$.

Interestingly, the replacement of $\mathrm{U}$ by $\psi$ in the STOP codons UAA, UAG, and UGA leads to nonsense suppression and incorporation of Ser, Thr for the first two and Tyr or Phe for the third STOP codon [21]. Crystal structures [22] show that the second codon base A is in syn and forms a cis Hoogsteen/Watson-Crick pair with G in the anticodon (the large dimension of such a pair is the same as in a Watson-Crick pair and therefore fits within the ribosomal grip of the A site $[23,24])$. However, the replacement of $U$ by $\psi$ in sense codon like UUU leads to a reduction of translation efficiency and without change in the nature of the amino acid inserted (especially at the second and third positions) [25]. Since the identification of 
pseudouridines in mRNAs [26, 27], the question of the effects of pseudouridylation on mRNA translation has been raised and discussed $[4,25,28]$. Up to now, the question is still pending and mysteries surrounding pseudouridines are still abound.

\section{Figure legends}

Figure 1: View of a pseudouridine in a helical conformation (PDB 3CGS). Carbon atoms are green, nitrogen atoms are blue and oxygen atoms are red. A water molecule (red sphere) links two anionic phosphate oxygens of the 5'-phosphate groups. Drawing made using Pymol (PyMOL(TM) 1.7.7.6 - Incentive Product Copyright (C) Schrodinger, LLC).

Figure 2: View of the 2'-5' linkage in the C complex of the spliceosome obtained after the first step of splicing that leads to the lariat formation [29]. The U2 is green, the branch point sequence is in pink and the bulged $\mathrm{A}$ forming the lariat is color-coded $(\mathrm{C}$ green, $\mathrm{N}$ blue, $\mathrm{O}$ red); it is followed by the two invariant first nucleotides of the intron GU that form a GU platform onto which the branched A stacks. The drawing made using Pymol is derived from the Pymol session on Kiyoshi Nagai's laboratory (cab be downloaded from https : / /www2 .mrc-lmb.cam.ac.uk/groups/nagai/resources/) • 


\section{References}

[1] S.D. Kennedy, W.J. Bauer, W. Wang, C.L. Kielkopf, Dynamic stacking of an expected branch point adenosine in duplexes containing pseudouridine-modified or unmodified U2 snRNA sites, Biochem Biophys Res Commun, 511 (2019) 416-421.

[2] J. Ge, Y.T. Yu, RNA pseudouridylation: new insights into an old modification, Trends Biochem Sci, 38 (2013) 210-218.

[3] F. Spenkuch, Y. Motorin, M. Helm, Pseudouridine: still mysterious, but never a fake (uridine)!, RNA Biol, 11 (2014) 1540-1554.

[4] X. Li, S. Ma, C. Yi, Pseudouridine: the fifth RNA nucleotide with renewed interests, Curr Opin Chem Biol, 33 (2016) 108-116.

[5] H. Jorjani, S. Kehr, D.J. Jedlinski, R. Gumienny, J. Hertel, P.F. Stadler, M. Zavolan, A.R. Gruber, An updated human snoRNAome, Nucleic Acids Res, 44 (2016) 5068-5082.

[6] D.R. Davis, Stabilization of RNA stacking by pseudouridine, Nucleic Acids Res, 23 (1995) 5020-5026.

[7] J.G. Arnez, T.A. Steitz, Crystal structure of unmodified tRNA(Gln) complexed with glutaminyl-tRNA synthetase and ATP suggests a possible role for pseudo-uridines in stabilization of RNA structure, Biochemistry, 33 (1994) 7560-7567.

[8] P. Auffinger, Westhof, E, Effects of pseudouridylation on tRNA hydration and dynamics: a theoretical approach, in: H. Grosjean, Benne, R (Ed.) Modification and Editing of RNA, ASM Press, Washington, D.C., 1996, pp. 103-112.

[9] Y. Lin, C.L. Kielkopf, X-ray structures of U2 snRNA-branchpoint duplexes containing conserved pseudouridines, Biochemistry, 47 (2008) 5503-5514.

[10] M.I. Newby, N.L. Greenbaum, Investigation of Overhauser effects between pseudouridine and water protons in RNA helices, Proc Natl Acad Sci U S A, 99 (2002) 12697-12702.

[11] F. Pochon, A.M. Michelson, M. Grunberg-Manago, W.E. Cohn, L. Dondon, Polynucleotide Analogues. Iii. Polypseudouridylic Acid: Synthesis and Some Physicochemical and Biochemical Properties, Biochim Biophys Acta, 80 (1964) 441-447.

[12] R. Parker, P.G. Siliciano, C. Guthrie, Recognition of the TACTAAC box during mRNA splicing in yeast involves base pairing to the U2-like snRNA, Cell, 49 (1987) 229-239.

[13] T.R. Mercer, M.B. Clark, S.B. Andersen, M.E. Brunck, W. Haerty, J. Crawford, R.J. Taft, L.K. Nielsen, M.E. Dinger, J.S. Mattick, Genome-wide discovery of human splicing branchpoints, Genome Res, 25 (2015) 290-303.

[14] M.I. Newby, N.L. Greenbaum, Sculpting of the spliceosomal branch site recognition motif by a conserved pseudouridine, Nat Struct Biol, 9 (2002) 958-965.

[15] E. Kierzek, M. Malgowska, J. Lisowiec, D.H. Turner, Z. Gdaniec, R. Kierzek, The contribution of pseudouridine to stabilities and structure of RNAs, Nucleic Acids Res, 42 (2014) 3492-3501.

[16] D.R. Davis, C.D. Poulter, 1H-15N NMR studies of Escherichia coli tRNA(Phe) from hisT mutants: a structural role for pseudouridine, Biochemistry, 30 (1991) 4223-4231.

[17] B. Kastner, C.L. Will, H. Stark, R. Luhrmann, Structural Insights into Nuclear premRNA Splicing in Higher Eukaryotes, Cold Spring Harb Perspect Biol, (2019).

[18] H. Grosjean, E. Westhof, An integrated, structure- and energy-based view of the genetic code, Nucleic Acids Res, 44 (2016) 8020-8040.

[19] F. Lecointe, O. Namy, I. Hatin, G. Simos, J.P. Rousset, H. Grosjean, Lack of pseudouridine 38/39 in the anticodon arm of yeast cytoplasmic tRNA decreases in vivo recoding efficiency, J Biol Chem, 277 (2002) 30445-30453. 
[20] K. Zerfass, H. Beier, Pseudouridine in the anticodon G psi A of plant cytoplasmic tRNA(Tyr) is required for UAG and UAA suppression in the TMV-specific context, Nucleic Acids Res, 20 (1992) 5911-5918.

[21] J. Karijolich, Y.T. Yu, Converting nonsense codons into sense codons by targeted pseudouridylation, Nature, 474 (2011) 395-398.

[22] I.S. Fernandez, C.L. Ng, A.C. Kelley, G. Wu, Y.T. Yu, V. Ramakrishnan, Unusual base pairing during the decoding of a stop codon by the ribosome, Nature, 500 (2013) 107-110.

[23] E. Westhof, Isostericity and tautomerism of base pairs in nucleic acids, FEBS Lett, 588 (2014) 2464-2469.

[24] E. Westhof, M. Yusupov, G. Yusupova, Recognition of Watson-Crick base pairs: constraints and limits due to geometric selection and tautomerism, F1000Prime Rep, 6 (2014) 19.

[25] T.P. Hoernes, N. Clementi, K. Faserl, H. Glasner, K. Breuker, H. Lindner, A.

Huttenhofer, M.D. Erlacher, Nucleotide modifications within bacterial messenger RNAs regulate their translation and are able to rewire the genetic code, Nucleic Acids Res, 44 (2016) $852-862$.

[26] T.M. Carlile, M.F. Rojas-Duran, B. Zinshteyn, H. Shin, K.M. Bartoli, W.V. Gilbert, Pseudouridine profiling reveals regulated $\mathrm{mRNA}$ pseudouridylation in yeast and human cells, Nature, 515 (2014) 143-146.

[27] S. Schwartz, D.A. Bernstein, M.R. Mumbach, M. Jovanovic, R.H. Herbst, B.X. LeonRicardo, J.M. Engreitz, M. Guttman, R. Satija, E.S. Lander, G. Fink, A. Regev,

Transcriptome-wide mapping reveals widespread dynamic-regulated pseudouridylation of ncRNA and mRNA, Cell, 159 (2014) 148-162.

[28] K. Kariko, H. Muramatsu, F.A. Welsh, J. Ludwig, H. Kato, S. Akira, D. Weissman, Incorporation of pseudouridine into mRNA yields superior nonimmunogenic vector with increased translational capacity and biological stability, Mol Ther, 16 (2008) 1833-1840. [29] W.P. Galej, M.E. Wilkinson, S.M. Fica, C. Oubridge, A.J. Newman, K. Nagai, Cryo-EM structure of the spliceosome immediately after branching, Nature, 537 (2016) 197-201. 
Figure 1

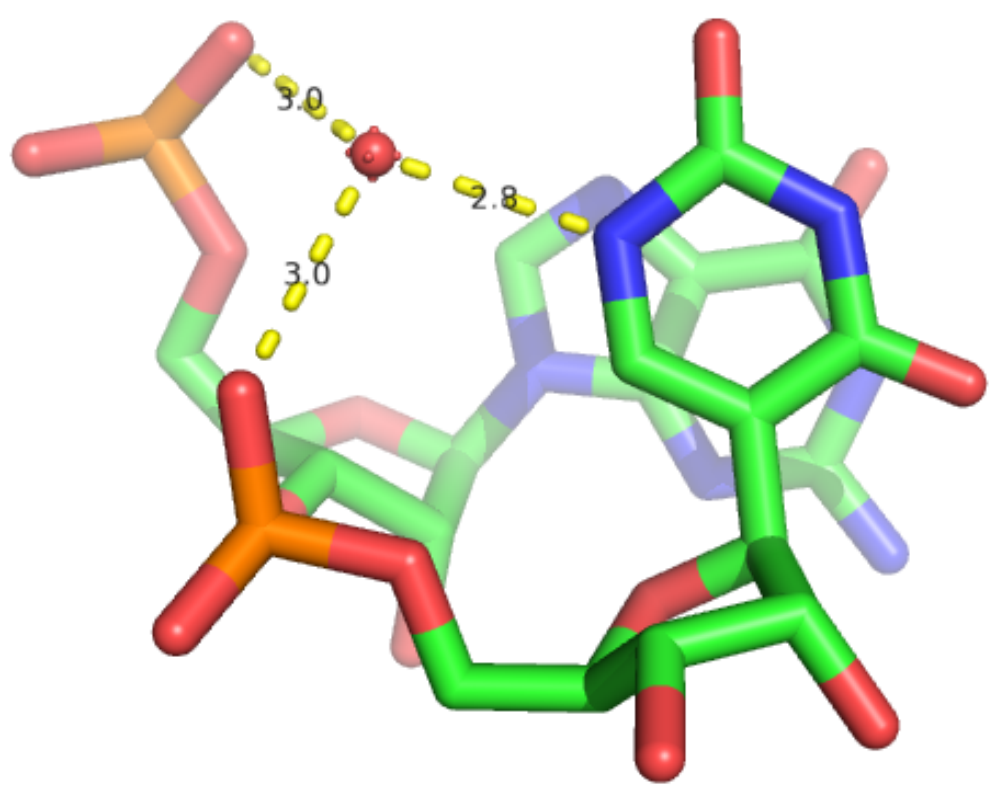


Figure 2

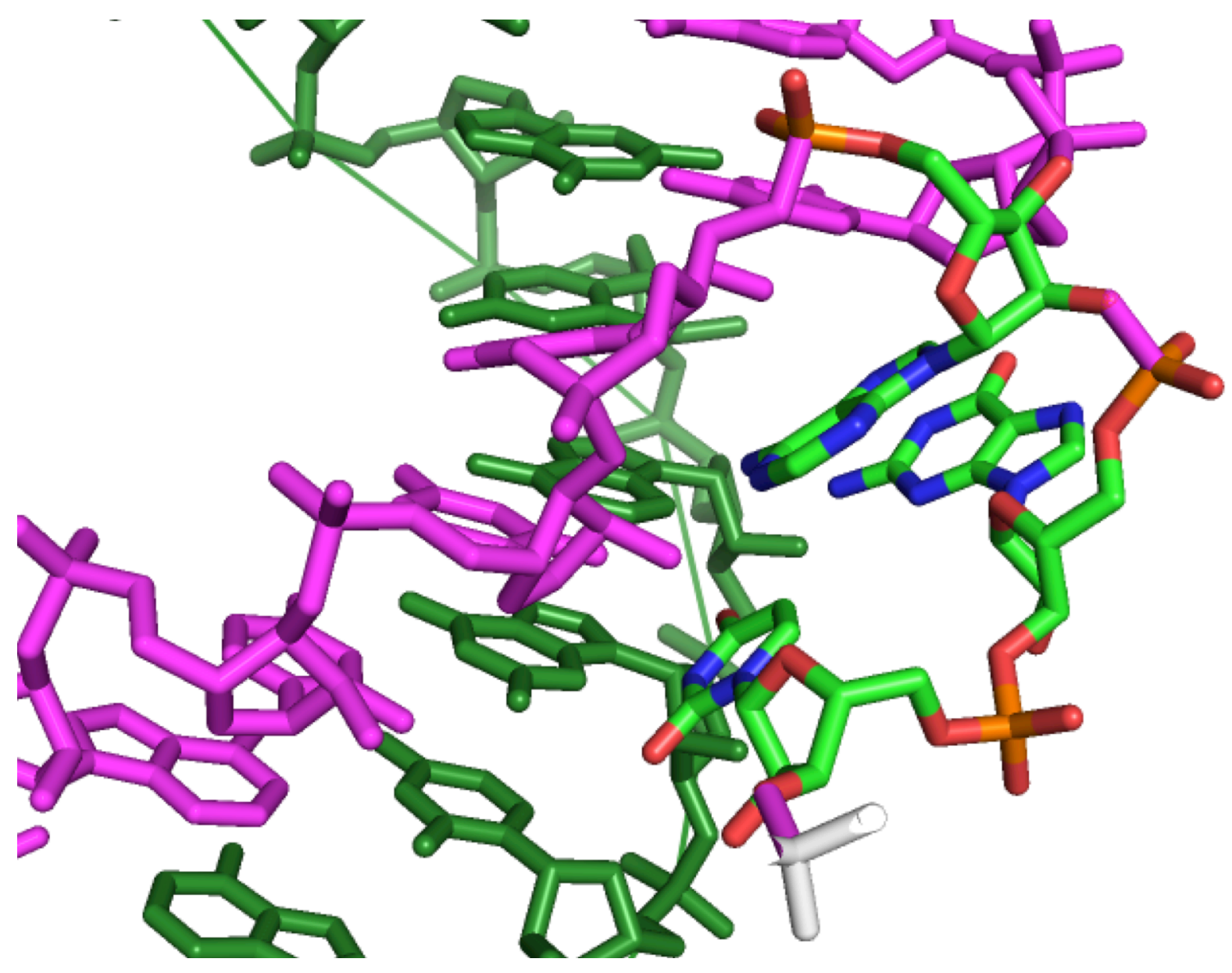


Figure 1

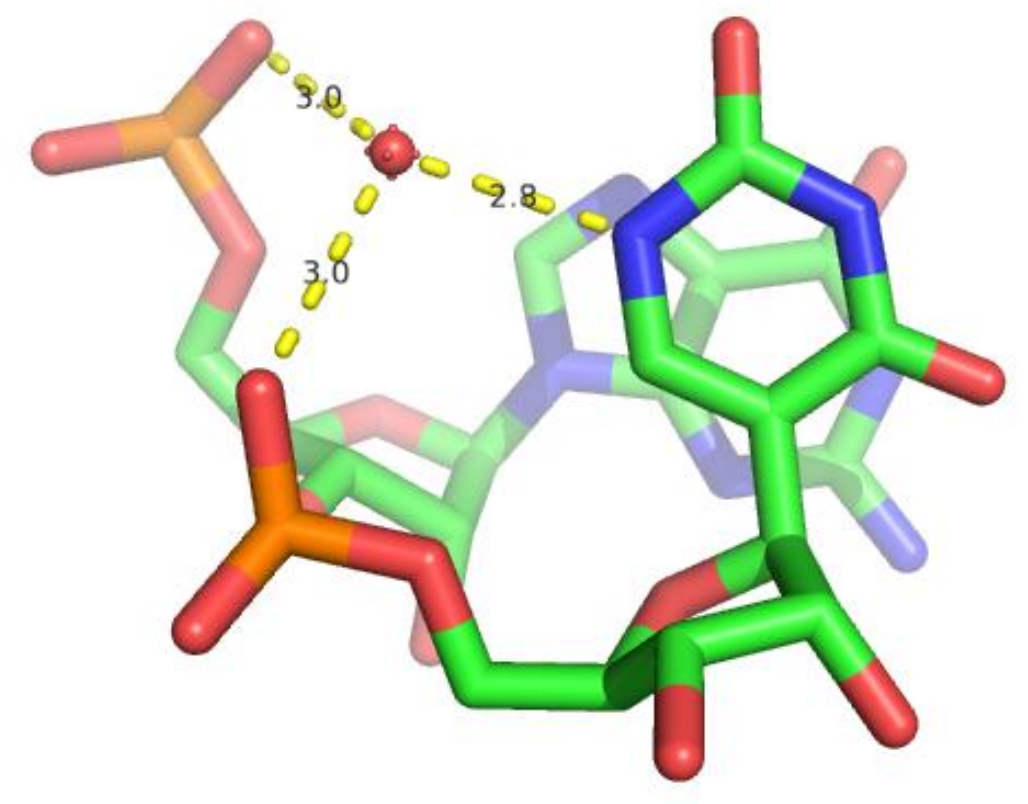

Figures 
Figure 2

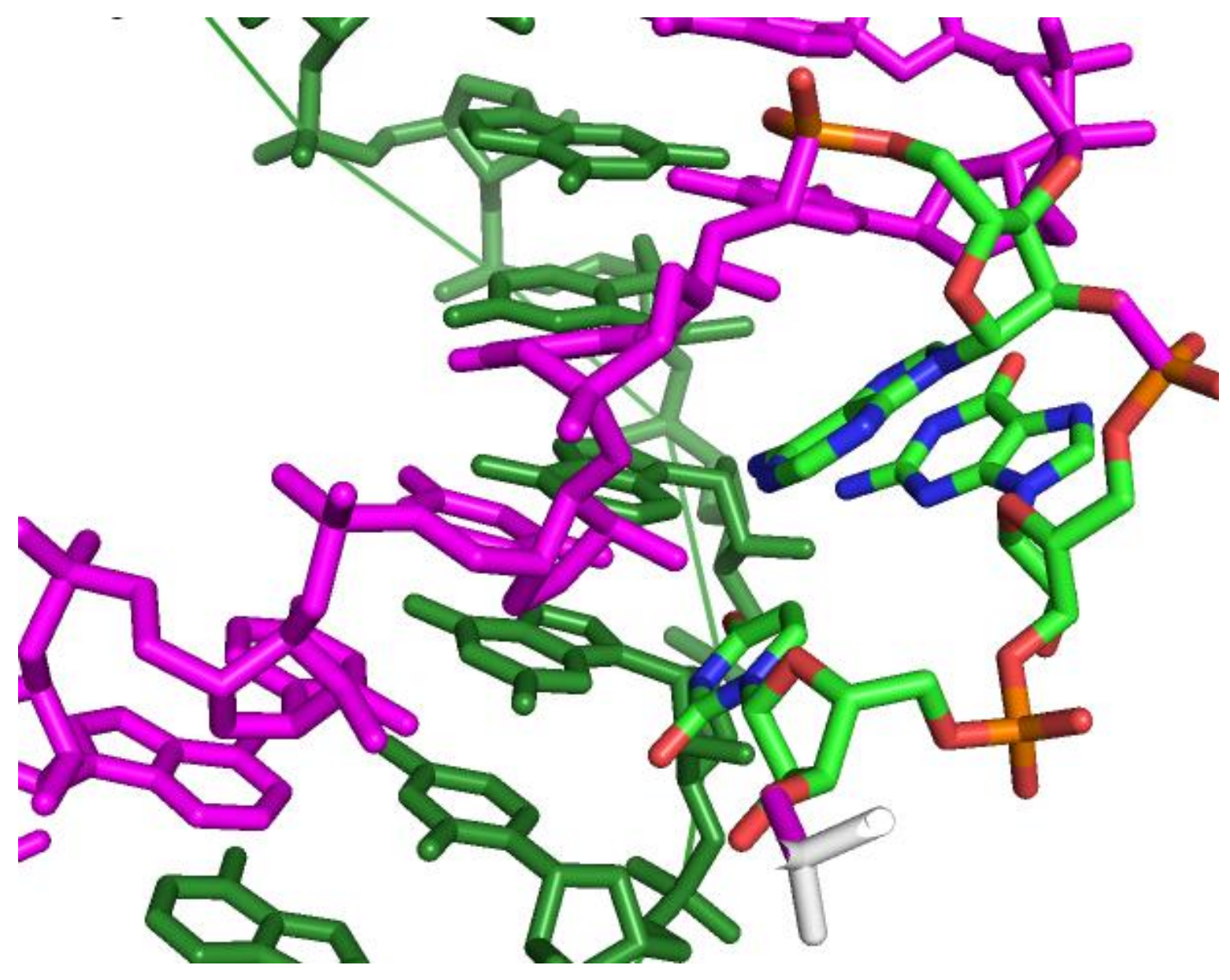

